\title{
Compromiso cardíaco en el síndrome de inmunodeficiencia adquirida. Primer reporte nacional
}

\author{
GERMÁN VALENZUELA ${ }^{1}$, ÓSCAR GUERRA² ${ }^{2}$, FRINE SAMALVIDES ${ }^{2}$, \\ RAÚL CASTILLO $^{1}$, FÉLIX MEDINA ${ }^{2}$, EDGAR GLORIA ${ }^{1}$ \\ ${ }^{1}$ Hospital Nacional Guillermo Almenara Irigoyen. Lima, Perú. \\ ${ }^{2}$ Hospital Nacional Cayetano Heredia. Lima, Perú.
}

\begin{abstract}
Resumen
Objetivo: Conocer los patrones de compromiso cardíaco en pacientes infectados por el virus de inmunodeficiencia humana en estadio SIDA. Material y Métodos: Estudio prospectivo de pacientes ambulatorios del Hospital Nacional Guillermo Almenara Irigoyen (HNGAI), que incluyó una evaluación de los parámetros clínicoepidemiológicos, así como una evaluación ecocardiográfica realizada entre marzo de 2002 y marzo de 2003. Resultados: Ciento treinta y dos pacientes fueron evaluados. Tuvieron 40,4 años en promedio (DE 8,7). La mediana del tiempo de diagnóstico fue 48 meses (RIQ 50) y recibieron terapia antiretroviral 63,6\%. Los patrones de compromiso cardíaco fueron agrupados en disfunción diastólica (DD) en 28,8\%, disfunción sistólica en $3,0 \%$, y derrame pericárdico en $9,1 \%$. La DD se asoció a una historia de internamientos anteriores así como a una mayor edad. Conclusiones: Encontramos un alto porcentaje de anormalidades cardíacas en pacientes ambulatorios con SIDA. La DD fue el hallazgo más frecuente.
\end{abstract}

Palabras clave: Síndrome de inmunodeficiencia adquirida; enfermedades cardiovasculares; ecocardiografía.

\begin{abstract}
Cardiac compromise in AIDS. First peruvian report Abstract

Objective: To determine the patterns of cardiac compromise in patients infected by human immunodeficiency virus in AIDS-stage. Material and Methods: Prospective study of ambulatory patients at Guillermo Almenara Irigoyen National Hospital between March 2002 and March 200, included assessment of clinical and epidemiological parameters and echocardiographic study. Results: One hundred and thirty-two patients were studied. They had a mean age of 40,4 years (SD 8,7). The median time for AIDS diagnosis was 48 months (IQR 50) and received antiretroviral therapy $63,6 \%$. Cardiac compromise patterns were diastolic dysfunction (DD) in $28,8 \%$, systolic dysfunction in $3,0 \%$, pericardial effusion in $9,1 \%$. DD was associated with a history of previous hospitalization and older age. Conclusions: We found a high percentage of cardiac abnormalities in patients with HIV infection in AIDS stage. DD was the most frequent finding.
\end{abstract}

Key words: Acquired inmunodeficiency syndrome; cardiovascular diseases; ectocrdiography.

\section{INTRODUCCIÓN}

El Síndrome de Inmunodeficiencia Adquirida (SIDA) se caracteriza por un estado profundo y adquirido de inmunosupresión, que predispone al paciente a infecciones por múltiples oportunistas, neoplasias malignas y a una disfunción progresiva de diferentes órganos y sistemas $\left({ }^{1-4}\right)$.

El compromiso cardíaco en pacientes con SIDA fue descrito por primera vez en
1983, por Austran, quien reportó sarcoma de Kaposi miocárdico en una autopsia. La prevalencia del compromiso cardíaco en pacientes con SIDA varía entre 28 y $73 \%$, dependiendo del tipo de estudio realizado $\left({ }^{1-3}\right)$.

La mejoría de la supervivencia de los pacientes con infección por el virus de inmunodeficiencia humana (VIH), relacionada de manera directa con el uso 
de terapia antiretroviral de alta eficacia (TARVAE); ha sido asociada a un incremento de complicaciones cardíacas. Al inicio de la epidemia, la enfermedad cardíaca fue considerada infrecuente, a pesar del elevado porcentaje de afección en los estudios de necropsias (25 a $75 \%)$ y ecocardiográficos (30 a 40\%), ya que ésta sólo presenta una clara traducción clínica en aproximadamente $10 \%$ de los pacientes infectados por el VIH $\left({ }^{1,3,5}\right)$.

La incidencia de la afección cardíaca en los pacientes con infección por el VIH varía ampliamente. Sin embargo, parece que surge comúnmente en los estadios clínicos avanzados de la enfermedad, cuando la inmunodepresión es mayor (generalmente, con cifras de linfocitos CD4< $200 \mathrm{cel} / \mathrm{uL}$ ), afectando a todos los grupos de riesgo $\left(^{3,6-9}\right)$.

Los hallazgos de compromiso cardíaco varían según el grupo poblacional evaluado, el estadio de infección, así como de acuerdo al empleo de terapia antiretroviral, historia de síndrome consuntivo asociado a VIH o infecciones por gérmenes oportunistas.

Con estas consideraciones, presentamos los resultados de la primera evaluación en nuestro país de pacientes con infección por VIH en estadio SIDA, realizada en el Hospital Nacional Guillermo Almenara Irigoyen, primer hospital nacional de la Seguridad Social en el país.

\section{MATERIAL Y MÉTODOS}

El diseño del estudio es prospectivo, descriptivo, de corte transversal en período. Los pacientes incluidos tuvieron infección por VIH-SIDA y fueron atendidos en el Hospital Nacional Guillermo Almenara Irigoyen. Se incluyó a pacientes adultos con infección por el virus de inmunodeficiencia humana (VIH) en estadio SIDA, que se atendían regularmente en los consultorios del hospital y que acudían a control médico ambulatorio.

Se excluyó a pacientes mayores de 60 años, con infección por VIH, en cualquier estadio diferente al estadio SIDA, portadores de enfermedad cardíaca congénita y con historia de enfermedades crónicas con compromiso cardiovascular (hipertensión arterial, diabetes mellitus, insuficiencia renal crónica, y alcoholismo).

Se consideró en estadio SIDA al paciente con infección por el virus de inmunodeficiencia humana en estadio clínico 4, según la clasificación de la OMS1990, o con niveles de linfocitos CD4 menores de $200 \mathrm{cel} / \mathrm{uL}$.

Se consideró en terapia antiretroviral a aquel paciente que tomaba medicamentos en los esquemas de terapia antiretroviral de alta eficacia (TARVAE) o esquemas alternativos, por un lapso mayor o igual a 16 semanas.

Se definió como patrón de disfunción diastólica la presencia de un cociente E/A $<1$, patrón normal si se encontraba entre 1 y 2,5 y patrón restrictivo si se encontraba mayor o igual de 2,5.

Los pacientes fueron seleccionados usando una tabla de números aleatorios. Posterior a su selección, fueron programados por el investigador principal para una evaluación ecocardiográfica, previa firma del consentimiento informado.

Dichos pacientes fueron evaluados siguiendo los parámetros de la ficha de recolección de datos, la cual incluía parámetros clínico-epidemiológicos relacionados con la infección por el VIH, así como parámetros ecocardiográficos. La evaluación ecocardiográfica se realizó en los consultorios de cardiología no invasiva. Un solo evaluador efectuó los estudios ecográficos, siguiendo los parámetros de evaluación preestablecidos.

Todas las mediciones fueron hechas con el equipo eco-doppler-color modelo HDI 
3500 de Phillips, siendo registradas en video. Se obtuvo proyecciones estándar (paraesternal izquierda, apical y subcostal) en decúbito lateral izquierdo y posición supina. Se analizó las siguientes variables ecocardiográficas: diámetros telesistólico y telediastólico del ventrículo izquierdo (VI), fracción de eyección (FE) y fracción de acortamiento (FA) del VI, dimensiones auriculares y presencia de derrame pericárdico.

Se utilizó el modo $M$ para las medidas del VI y la fórmula de Teicholz para el cálculo de la FE y FA. Se obtuvo los parámetros de flujos diastólicos con Doppler pulsado situando la muestra entre ambas valvas de la válvula mitral, y todos los valores fueron promediados en dos ciclos cardíacos consecutivos. Las variables registradas fueron: velocidad máxima de onda $\mathrm{E}$ y onda $\mathrm{A}$, relación onda E/A, y tiempo de relajación isovolumétrica $\left({ }^{10,11}\right)$.

Para el análisis estadístico, las variables cuantitativas fueron presentadas como media y desviación estándar y las variables cualitativas en porcentajes. Para el caso del hallazgo ecocardiográfico de disfunción diastólica del ventrículo izquierdo, se empleó estadística bivariada, comparando

Tabla 1. Características clínico-epidemiológicas.

\begin{tabular}{lrr}
\hline \multicolumn{1}{l}{$\mathrm{n}$} & $\%$ \\
\hline Sexo & & \\
Masculino & 84 & 63,6 \\
Femenino & 48 & 36,4 \\
Grupos de edad (años)* & & \\
$<20$ & 0 & 0,0 \\
$20-30$ & 18 & 13,6 \\
$30-40$ & 56 & 42,4 \\
$40-50$ & 36 & 27,3 \\
$50-60$ & 22 & 16,7 \\
Tiempo de diagnóstico & & \\
$<1$ año & 26 & 19,7 \\
$1-5$ año & 64 & 48,5 \\
$5-10$ años & 38 & 28,8 \\
$>10$ años & 4 & 3,0 \\
\hline
\end{tabular}

*son eventos no excluyentes su presencia en función a otras variables clínicas o de laboratorio, así como estadística multivariada con el método de regresión logística entre variables clínicas asociadas a disfunción diastólica. El análisis fue realizado con el programa SPSS versión 10,0.

\section{RESULTADOS}

Ciento treinta y dos pacientes fueron evaluados entre los meses de marzo de 2002 y marzo de 2003. La edad promedio de los pacientes evaluados fue 40,4 años (DE 8,7), siendo de sexo masculino 84 pacientes $(63,6 \%)$. El grupo de edad se ubicó entre los 30 y 40 años de edad $(42,4 \%)$, en menor proporción en el grupo de 40 a 50 años $(27,3 \%)$ y en el grupo entre 50 y 60 años $(16,7 \%)$ (Tabla 1$)$.

La media del tiempo de diagnóstico fue 49,1 meses (DE 33,9). Al desdoblarse esta variable en subgrupos, se encontró que 32 pacientes tuvieron un tiempo de diagnóstico entre 1 y 5 años $(48,5 \%), 38$ pacientes $(28,8 \%)$ entre 5 y 10 años de diagnóstico, mientras que sólo 4 pacientes tuvieron más de 10 años $(3,0 \%)$.

Respecto al empleo de medicación antiretroviral, se encontró que $36,4 \%$ de

Tabla 2. Condición definidora de SIDA.

\begin{tabular}{lrr}
\hline Condición definidora de SIDA & $\mathrm{n}$ & \multicolumn{1}{c}{$\%$} \\
\hline Síndrome consuntivo & 36 & 27,3 \\
TBC pulmonar/extrapulmonar & 18 & 13,6 \\
Neumonía por P.carinii & 12 & 9,1 \\
MEC criptococócica & 2 & 1,5 \\
Candidiasis esofágica & 4 & 3,0 \\
Toxoplasmosis cerebral & 0 & 0 \\
Infección por citomegalovirus & 0 & 0 \\
Criptosporidiasis & 0 & 0 \\
Linfoma & 0 & 0 \\
CD4 menor de 200 & 54 & 40,9 \\
Neumonía recurrente & 2 & 1,5 \\
Leucoencefalopatía multifocal & 2 & 1,5 \\
Isosporidiasis & 2 & 1,5 \\
\hline
\end{tabular}


los pacientes se encontraba sin terapia, recibiendo todos los casos restantes terapia antiretroviral de alta eficacia.

La condición definidora de SIDA fue predominantemente el hallazgo de laboratorio del recuento de linfocitos CD4 menores de $200 \mathrm{cel} / \mathrm{uL}$ en pacientes asintomáticos, pero con factores de riesgo, que fueron derivados a los consultorios externos respectivos $(40,9 \%)$. Sin embargo, también se registraron algunas condiciones definidoras asociadas, como el síndrome de consumo asociado a SIDA, el cual se presentó en 36 pacientes

Tabla 3. Compromiso cardíaco en SIDA.

Hallazgos globales de compromiso cardíaco

\begin{tabular}{lrr}
\hline Alteración & $\mathrm{n}$ & $\%$ \\
\hline Derrame pericárdico & 12 & 9,1 \\
Disfunción sistólica & 4 & 3,0 \\
Disfunción diastólica & 38 & 28,8 \\
Cardiopatía restrictiva & 0 & 0,0 \\
Hipertensión pulmonar & 0 & 0,0 \\
Pericarditis constrictiva & 0 & 0,0
\end{tabular}

$(27,3 \%)$.

El diagnóstico de SIDA fue hecho también, aunque en porcentajes menores,

Tabla 4. Parámetros asociados a disfunción diastólica.

\begin{tabular}{lcc}
\hline Parámetros & $\begin{array}{r}\mathrm{DD} \\
(\mathrm{n}=38)\end{array}$ & $\begin{array}{c}\text { No DD } \\
(\mathrm{n}=94)\end{array}$ \\
\hline Edad (años) & 42,6 & $38,8 *$ \\
Tiempo de diagnóstico(meses) & 32,3 & 35,2 \\
Recuento de CD4 & 160,8 & 166,7 \\
Terapia antiretroviral & $14 / 38$ & $39 / 94$ \\
-Inhibidores de proteasa & $12 / 38$ & $36 / 94$ \\
Internamientos anteriores & $14 / 38$ & $18 / 94 *$ \\
Pérdida de peso & $10 / 38$ & $25 / 94$ \\
Fracción de eyección $(\%)$ & 62,4 & 61,4 \\
Fracción de acortamiento $(\%)$ & 36,8 & 35,2 \\
\hline$* p<0,05$ & &
\end{tabular}

por la presencia de infecciones por gérmenes oportunistas asociadas. Las más importantes fueron la TB pulmonar o sistémica en 18 pacientes $(13,6 \%)$ y la neumonía por Pneumocystis carinii en 12 pacientes $(9,1 \%)$. En porcentajes menores, se encontró criptococosis meningoencefálica, candidiasis esofágica y toxoplasmosis cerebral. Cabe resaltar que la presencia de coinfecciones al diagnóstico de SIDA se registró en $20 \%$ de los casos evaluados (Tabla 2).

Los hallazgos ecocardiográficos sugerentes de disfunción diastólica del ventrículo izquierdo, fueron los hallazgos preponderantes, encontrándose en $28,8 \%$ de casos, respectivamente (Tabla 3 ). También se encontró a 12 pacientes con derrame pericárdico, todos de grado levemoderado $(9,1 \%)$ y 4 pacientes con disfunción sistólica del ventrículo izquierdo $(3,0 \%)$. La fracción de eyección promedio en este grupo de pacientes fue de $37 \%$.

Como la disfunción diastólica fue el hallazgo ecocardiográfico más frecuentemente encontrado, decidimos estudiar los parámetros clínicos y de laboratorio asociados. Mediante análisis bivariado, se reconoció que las variables asociadas a este hallazgo fueron el tener una mayor edad media, así como una historia de internamientos anteriores. Por otro lado, al evaluarse los parámetros clínicos asociados a la presencia de disfunción diastólica mediante los métodos Enter y Forward-Wald de regresión logística, se encontró que la única variable relacionada con la presencia de disfunción diastólica fue la edad $(p=0,005)$.

\section{DISCUSIÓN}

El corazón ha sido el órgano menos estudiado en el grupo de pacientes con infección por el VIH, probablemente por su incierta repercusión clínica y porque su compromiso fisiopatogénico no está claro 
aún.

Hace dos décadas se comenzó a describir algunas anormalidades ecocardiográficas en pacientes infectados. Estos estudios inicialmente incluyeron un pequeño número de casos y fueron dirigidos por cardiólogos, lo cual condicionó la ausencia de registro de parámetros referidos a la infección por el VIH. Posteriormente, grupos conformados por médicos de diferentes especialidades consiguieron evaluar a un mayor número de pacientes; tratando en algunas oportunidades de identificar al agente causal de la infección en el tejido miocárdico, o a patógenos involucrados en la génesis de infecciones oportunistas condicionadas por el estado de inmunosupresión $\left.{ }^{(12-21}\right)$.

A pesar de estos reportes, los estudios clínicos en pacientes asintomáticos en condiciones severas de inmunosupresión son escasos a nivel mundial, siendo en nuestro país el primer estudio realizado.

Encontramos que nuestro grupo estudiado incluyó a una población joven de pacientes asegurados, con una edad promedio de 40 años, en los cuales el diagnóstico de SIDA se realizó predominantemente por conteos de CD4 bajos y que recibían terapia antiretroviral de alta eficiacia, en un elevado porcentaje de casos, dado que la distribución de medicación antiretroviral en el Seguro Social de Salud peruano es gratuita, al haberse evaluadoíntegramente al paciente y haberse establecido las posibilidades benéficas de esta estrategia farmacológica. En porcentajes menores, el diagnóstico de SIDA fue hecho por la presencia de más de una infección por gérmenes oportunistas.

Los datos de alteración de la relajación del ventrículo izquierdo sugestivos de disfunción diastólica, se constituyeron en los hallazgos preponderantes en nuestro grupo de pacientes $(28,8 \%)$, porcentaje mayor que el reportado en la literatura mundial $\left(^{1-4,22}\right)$. En la mayoría de los casos, se presentaron sin encontrarse asociados ni a la presencia de derrame pericárdico ni a la presencia de hipertensión pulmonar significativa, u otras alteraciones. Este grupo de pacientes fue de mayor edad y con una historia de más de dos internamientos anteriores.

Respecto a la asociación de infecciones oportunistas y compromiso cardíaco en pacientes con infección por VIH; está probado que algunas infecciones de manera directa e indirecta lesionan el miocito cardíaco, condicionando manifestaciones de enfermedad cardíaca $\left({ }^{23-27}\right)$.

El hallazgo del compromiso mayor de función linfocitaria y el compromiso de la función diastólica sugiere una posible afectación miocárdica de naturaleza inmune. En los modelos de miocarditis, que es la causa mejor estudiada de cardiomiopatía dilatada en enfermedad por VIH, los viriones infectan las células miocárdicas en parche, sin una clara asociación entre el VIH y la disfunción del miocito cardíaco. El ingreso del virus a células CD4 negativas es desconocido, propugnándose la participación de células de reservorio, como las dendríticas, o la activación de citoquinas multifuncionales, como TNF, IL-1, IL-6 e IL-10. Se sabe bien que el TNF-alfa produce un efecto inotrópico negativo, alterando la homeostasis de calcio e induciendo la síntesis de óxido nítrico, con efecto similar sobre la contractibilidad del miocito $\left({ }^{3,14,28}\right)$. Por otro lado, algunos virus cardiotropos (Coksackie virus del grupo B, citomegalovirus) han sido relacionados con el desarrollo de una miocardiopatía de posible origen viral en pacientes con recuentos de CD4s menores de $200\left(^{(29-35}\right)$.

Reportes recientes han evaluado el riesgo incrementado de aparición de enfermedad coronaria con el uso de inhibidores de proteasa $\left(^{66,37}\right)$. También se reporta la asociación de su uso con la presencia de cambios ventriculares y de 
disfunción diastólica. El grupo de Meng y colaboradores evaluó a 98 pacientes, 55 en tratamiento con inhibidores de proteasa y 43 sin ese esquema; encontrándose diferencias entre las razones de las ondas E/A entre ambos grupos. Esta razón es un claro marcador de la presencia de disfunción diastólica y estuvo asociada con la duración de la terapia por más de 1 año $\left.{ }^{(38}\right)$ Por el contrario, nuestros datos no encontraron asociación entre disfunción diastólica y terapia antiretroviral, con o sin inhibidores de proteasa $\left({ }^{39-40}\right)$.

El derrame pericárdico se presentó como la segunda alteración más frecuente en nuestra serie $(9,1 \%)$, porcentaje mucho menor que el reportado en otros estudios, donde fue en promedio $25 \%$. El compromiso pericárdico es una de las formas más comunes de compromiso cardiovascular en la infección por el VIH, existiendo una gran variedad de manifestaciones clínicas, dentro de las cuales se incluyen efusión pericárdica asintomática, pericarditis, taponamiento cardíaco y pericarditis constrictiva. Hasta ahora no hay acuerdo sobre la etiología del derrame pericárdico en la infección por el VIH. Un origen infeccioso pudiera ser el más frecuente, con amplias variaciones geográficas, pero diversos estudios que incluyen pericardiocentesis, biopsia pericárdica y cultivo mostraron resultados no concluyentes, por lo cual su utilidad deberá analizarse de acuerdo al contexto clínico de cada paciente $\left({ }^{41,42}\right)$.

La prevalencia de disfunción sistólica de $3,0 \%$ en nuestra serie fue baja en relación con otros reportes mundiales, y se ha asociado a una manifestación tardía de afectación cardíaca en la infección por el VIH; sin embargo, si se analiza las anormalidades de la función contráctil por segmentos, este porcentaje podría aumentar $\left({ }^{43-45}\right)$. En nuestro estudio. estos casos no fueron secundarios a cardiomiopatía dilatada, cuya prevalencia se reporta en otras latitudes entre el 10 y $30 \%$, asociándose a un tiempo de supervivencia mucho menor.

No se encontró casos de hipertensión pulmonar (HTP). Sin embargo, este hallazgo ha sido reportado en pacientes con infección por el VIH, donde la tasa de HTP es 100 veces mayor, siendo los factores de riesgo más comunes el uso de drogas endovenosas, contactos homosexuales y hemofilia $(1,3,46)$.

Nuestro estudio encontró un considerable porcentaje de anormalidades cardíacas detectadas por el examen ecocardiográfico, en pacientes adultos jóvenes con infección VIH-SIDA. En este grupo de pacientes, la disfunción diastólica relacionada con un patrón ecocardiográfico anormal de relajación del ventrículo izquierdo fue el hallazgo más común y estuvo relacionado con una mayor edad y el antecedente de pérdida de peso.

Por ello recomendamos el examen clínico rutinario del corazón en pacientes con infección por virus de inmunodeficiencia humana, especialmente para aquellos en condiciones severas de inmunosupresión. El método de ayuda diagnóstica complementaria es el estudio ecocardiográfico, con énfasis en los parámetros ya mencionados, y debería realizarse al momento del diagnóstico de la infección por VIH y luego 1 vez por año para pacientes sin patologías cardíacas estructurales o funcionales; $y$, a intervalos más cortos para pacientes con hallazgos anormales.

\section{AGRADECIMIENTOS}

Al Concejo Nacional de Ciencia y Tecnología por el apoyo brindado para el desarrollo y publicación del presente estudio.

\section{REFERENCIAS BIBLIOGRÁFICAS}

1. Rerkpattanapipat P, Wongpraparut N, Jacobs L, Kotler M. Cardiac manifestations of acquired immunodeficiency syndrome. Arch Intern Med. 2000;160:602-8. 
2. Grody W, Cheng L, Lewis W. Infection of the heart by the human immunodeficiency virus. Am J Cardiol. 1990;66:2036.

3. Flotals A, Domingo P, Carrió I. Afección cardíaca en la infección por el virus de la inmunodeficiencia humana. Med Clin. 1999;113:383-90.

4. Currie P, Jacob A, Foreman A. Heart muscle disease related to HIV infection: prognostic implications. BMJ. 1994;309:1605-7.

5. Valdez H, Chowdhry T, Assad R, Woolley. Changing spectrum of mortality due to Human Inmunodeficiency Virus: analysis of 260 deaths during 1995-1999. Clin Infect Dis. 2001;32:1487-93.

6. Lobasco G, Tinoco E, Martins L, Ribeiro M. Doppler echocardiographic evaluation of HIV-positive patients in different stages of the disease. Arq Bras Cardiol. 1999;73:163-8.

7. Hernández F, Gascueña R, Escribano P, Velásquez M. Disfunción diastólica en la infección por el virus de la inmunodeficiencia humana. Rev Esp Cardiol. 2001;54:11839.

8. Longo-Mbenza B, Seghers KV , Phuati M, Bikangi N. Heart involvement and HIV infection in african patients. Determinants of survival. Int J Cardiol. 1998;64:63-73.

9. Longo-Mbenza B, Seghers LV, Kintoki E. Assessment of ventricular diastolic function in AIDS patients from Congo: a Doppler echocardiographic study. Heart. 1998;80:184-9.

10. Sahn D, De Maria A, Kisslo J, Weyman A. Recommendations regarding quantitation in M-mode echocardiography: results of a survey of echocardiographic measurements. Circulation. 1978;58:1072-82.

11. Devereux R, Reichek N. Echocardiographic determination of left ventricular mass in man. Circulation. 1977;55:613-8.

12. Silva-Cardoso T, Moura B, Martins L, Mota-Miranda A, Rocha-Goncalves F, Lecour H, et al. Pericardial involvement in human immunodeficiency virus infection. Chest. 1999;115:418-22.

13. Barbaro G, Di Lorenzo G, Grisorio B, Barbarini G. Incidence of dilated cardiomyopathy and detection of HIV in myocardial cells of HIV-positive patients. N Engl J Med. 1998;339:1093-9.

14. Shannon R, Simon M, Mathler M, Geng Y, Mankaa S, Lackner A, et al. Dilated cardiomyopathy associated with simian AIDS in nonhuman primates. Circulation. 2000;101:185-93.

15. Sánchez J, Mazzoti G, Cuéllar L, Campos P, Gotuzzo E. SIDA. Epidemiología, diagnóstico, tratamiento y control de la infección VIH/SIDA. 1era ed. Lima: OMS; 1994.

16. Levy W, Simon G, Ríos J, Ross A. Prevalence of cardiac abnormalities in human immunodeficiency virus infection. Am J Cardiol. 1989;63:86-9.

17. Heidenreich P, Eisenberg M, Kee L, Somelofski C, Hollander $\mathrm{H}$, Schiller N, et al. Pericardial effusion in AIDS. Circulation. 1995;92:3229-34.

18. Lai W, Lipshultz S, Easley K, Starc T. Prevalence of congenital cardiovascular malformations in children of human immunodeficiency virus-infected women. The prospective P2C2 HIV multicenter study. J Am Coll Cardiol. 1998;32:1749-55.

19. Lipshultz S, Easley K, Orav J, Kaplan S. Left ventricular structure and function in children infected with human immunodeficiency virus. The prospective P2C2 HIV multicenter study. Circulation. 1998;97:1246-56.

20. Eisenhauer M, Eliasson A, Taylor A, Coyne O, Wortham D. Incidence of cardiac arrhytmias during intravenous pentamidine therapy in HIV-infected patients. Chest. 1994;105:389-94.

21. Girgis I, Gualberti J, Langan L, Malek S. A prospective study of the effect of IV pentamidine therapy on ventricular arrhythmias and QTc prolongation in HIV-infected patients. Chest. 1997;112:646-53.

22. Himelman RB, Chung WS, Chernoff DN, Schiller NB, Hollander H. Cardiac manifestations of human immunodeficiency virus infection: a two dimensional echocardiographic study. J Am Coll Cardiol. 1989;13:10306.

23. Grant A, M de Cock K. HIV infection and AIDS in the developing world. BMJ. 2001;322:1475-8.

24. Bárbaro G, Barbarini G, Di Lorenzo G. Early impairment of systolic and diastolic function in asymptomatic HIV-positive patients: a multicenter echocardiographic and echo-doppler study. AIDS Research and Human Retroviruses. 1996;12:1559-63.

25. Martínez-García T, Sobrino JM; Pujol E, Galvez J, Benitez E, Girón-González JA. Ventricular mass and diastolic function in patients infected by the human inmunodeficiency virus. Heart. 2002;84:620-4.

26. Monsuez JJ, Kinney EL, Vittecoq D, Kitzis M, Rozembaum W, D'Agay MF, et al. Comparison among acquired immune deficiency syndrome patients with and without clinical evidence of cardiac disease. Am J Cardiol. 1998;62:1311-3.

27. Barbaro G. Cardiovascular manifestations of HIV infection. Circulation. 2002;106:1420-5.

28. Reilly J, Cunnion R, Anderson D, O’Leary, et al. Frequency of myocarditis, left ventricular dysfunction and ventricular tachycardia in the Adquired Immune Deficiency Syndrome. Am J Cardiol. 1988;62:789-93.

29. Barbaro G, Di Lorenzo G, Soldini M. Intensity of myocardial expression of inducible nitric oxide synthase influences the clinical course of human immunodeficiency virus-associated cardiomyopathy. Circulation. 1999;100:933-9.

30. St John Sutton M, Plappert T, Crosby L, Douglas P, Mullen J, Reichek N. Effects of reduced left ventricular mass on chamber architecture, load, and function: a study of anorexia nervosa. Circulation. 1985;72:991-1000.

31. Gottdiener J, Gross H, Henry W, Borer J, Ebert M. Effects of self-induced starvation on cardiac size and function in anorexia nervosa. Circulation. 1978;58:425-33.

32. Mac Mahon S, Wilcken D, Mac Donald G. The effect of weight reduction on left ventricular mass. N Engl J Med. 1986;314:334-9.

33. Alpert M, Lambert C, Terry B, Kelly D. Effect of weight loss on left ventricular mass in nonhypertensive morbidly obese patients. Am J Cardiol. 1994;73:918-21.

34. Alpert M, Terry B, Lambert C, Kelly D. Factors influencing left ventricular systolic function in non hypertensive morbidly obese patients, and effects of weight loss induced by gastroplasty. Am J Cardiol. 1993;71:733-7.

35. Anker S, Ponikowski P, Varney S. Wasting as independent 
1997;349:1050-3

36. Holmberg SD, Moorman AC, Williamson JM; Tong TC, Ward DJ, Wood KC, et al. Protease inhibitors and cardiovascular outcomes in patients with HIV-1. Lancet. 2002;360:1747-8.

37. Klein D, Hurley LB, Quesenberry CP, Sidney S. Do protease inhibitors increase the risk for coronary heart disease in patients with HIV-1 infection?. JAIDS. 2002;30:471-7.

38. Meng Q, Lima JA, Lai H, Vlahow D, Celentano DD, Strathdee $\mathrm{S}$, et al. Use of HIV Protease inhibitors is associated with left ventricular morphologic changes and diastolic dysfunction. JAIDS. 2002;30:306-10.

39. Lipshultz S, Easley K, Oray J, Kaplan S. Absence of cardiac toxicity of zidovudine in infants. N Engl J Med. 2000;343:759-66.

40. Neild P, Amadi A, Ponikowski P, Coats A, Gazzard B. Cardiac autonomic dysfunction in AIDS is not secondary to heart failure. Int J Cardiol. 2000;74:133-7.

41. Kwan T, Karve M, Emerole O. Cardiac tamponade in patients infected with HIV. Chest. 1993;104:1059-62.
42. Gouny O, Lancelin C, Girard P. Pericardial effusion and AIDS: benefits of surgical drainage. Eu J Cardio-thoracic Surg. 1998;13:165-9.

43. Herskowitz A, Vlahow D, Willoghby S, Chaisson R. Prevalence and incidence of left ventricular dysfunction in patients with human immunodeficiency virus infection. Am J Cardiol. 1993;71:955-8.

44. Willoghby S, Vlahov D, Herskowith A. Frequency of left ventricular dysfunction and other echocardiographic abnormalities in human immunodeficiency virus seronegative intravenous drug users. J Am Coll Cardiol. 1993;71:446-7.

45. Umana E, Panayiotou H, Ramsey K, Bikkina M, Massey C, Alpert M. Left ventricular mass and systolic function in human immunodeficiency virus-infected patients after weight loss. Am J Cardiol. 2000;86:1040-3.

46. Petipretz P, Brenot F, Azarian R. Pulmonary hypertension in patients with human immunodeficiency virus infection. Circulation. 1994; 89:2722-7.

\author{
Correspondencia: \\ Dr. Germán V. Valenzuela \\ Hospital Nacional Edgardo Rebagliati Martins \\ Servicio de Cardiología (piso 118) \\ Av. Salaverry $s / n$ \\ Lima 11, Perú \\ Correo-e: valenzuela_german@yahoo.es
}

